\title{
Folding transitions of the triangular lattice with defects
}

\author{
Emilio N.M. Cirillo and Giuseppe Gonnella \\ Dipartimento di Fisica dell'Università di Bari and \\ Istituto Nazionale di Fisica Nucleare, Sezione di Bari \\ via Amendola 173, 70126 Bari, Italy \\ Alessandro Pelizzola \\ Dipartimento di Fisica del Politecnico di Torino and \\ Istituto Nazionale per la Fisica della Materia \\ c. Duca degli Abruzzi 24, 10129 Torino, Italy
}

\begin{abstract}
A recently introduced model describing the folding of the triangular lattice is generalized allowing for defects in the lattice and written as an Ising model with nearestneighbor and plaquette interactions on the honeycomb lattice. Its phase diagram is determined in the hexagon approximation of the cluster variation method and the crossover from the pure Ising to the pure folding model is investigated, obtaining a quite rich structure with several multicritical points. Our results are in very good agreement with the available exact ones and extend a previous transfer matrix study.
\end{abstract}

PACS numbers: 05.50.+q (Ising problems); 64.60.-i (General studies of phase transitions); 82.65.Dp (Thermodynamics of surfaces and interfaces). 


\section{Introduction}

Polymerized membranes are two-dimensional generalizations of linear polymers; the large variety of possible applications and the extension of one-dimensional statistical properties to $2 D$ objects justifies recent numerous studies on these systems [1, 2, 6, 4, 5, 6, 7, 8]. Differently from linear polymers, polymerized membranes are expected to show different long-distance behaviours depending on the microscopic characteristics of the system. In particular, rigid polymerized membranes are expected to be stable in a flat phase [3] which has no analogue in polymer systems.

Models of polymerized membranes consist of networks with fixed connectivity fluctuating in an embedding space. The length of the bonds can vary with an energy cost diverging for increasing lengths, while the rigidity of the network is described by a bending energy term favouring flat configurations. When excluded volume effects are not considered, as it will be in the model of this paper, it has been shown [2, 3, 4, 5, 6, 8] that by varying the strength $K$ of the bending term, a critical transition arises separating a flat phase at large $K$ from a crumpled phase at small $K$.

The case of a triangular network embedded in a $d$-dimensional space where the bonds are of fixed length has been first considered in [9]. Here the only degrees of freedom correspond to the possible ways of folding the network. In [9] the problem has been studied in a $2 d$ embedding space. In this simplified case the normal vectors to the triangles point either up or down in some direction, suggesting a description of the system in terms of Ising spin variables. The entropy of this folding problem has been recognized [10 to be the same entropy of the problem of colouring with three colours the bonds of the hexagonal lattice, 
which has been exactly calculated in [11]. In the description of the model in terms of Ising variables a bending rigidity can be easily defined [9]; in [12] a first-order $2 d$ folding transition between a crumpled and a flat phase has been found at a finite value of the bending strength $K$.

In this paper we consider again the $2 d$ folding problem of a triangular network; we apply the cluster variation method (CVM) [13, 14, 15] to complete the study of [12] analyzing the phase diagram of the model in the whole plane $K, h$ where $h$ is a symmetry breaking field distinguishing between up and down triangles. The folding problem can be expressed as a vertex model or an Ising problem with a local constraint on the possible spin configurations [10]. We have also studied the system by progressively relaxing the constraint until the standard Ising model is obtained. The relaxing of the constraint corresponds to accept folding configurations with endpoints in the folding lines, that is cuts between adjacent triangles of the network. Therefore intermediate models between the Ising model and the folding model of [9, 10, 12] could describe realistic polymerized membranes where defects in the connectivity rules are present.

The cluster variation method is known to be very accurate in describing the phase diagram of magnetic systems [16]. Also in this case of a constrained magnetic system, the CVM gives an accurate description of the system. For example, the $T=\infty$ entropy calculated with the CVM is $\ln q, q=\sqrt{13} / 3 \approx 1.2019$ which has to be compared with the exact value $q=1.2087$ [11]. In the whole plane $K, h$, in addition to the first order transition between the flat and the folded disordered phase [12], we have found at negative $K$ a critical transition between the disordered phase and a folded phase with staggered antiferromagnetic order. When the 
constraint is progressively relaxed the topology of the phase diagram is found to evolve in quite a complex way, resembling at negative $K$ the phase diagram of the Ising model for metamagnets [17], until the standard Ising model phase diagram is recovered.

The paper is organized as follows. In section 2 the model of folding is defined in terms of spin variables and the CVM approximation scheme used for studying the phase diagram is briefly described. In section 3 the phase diagrams of the folding system with the constraint progressively relaxed are given. In section 4 our results will be briefly discussed.

\section{The model and the method}

We briefly describe the model of folding studied in [9, 10, 12]. In a $2 d$ embedding space the normal vectors to the triangles of a regular network can be represented by Ising spins $s_{i}= \pm 1$, where $i$ denotes a site of a honeycomb lattice (the dual of the triangular lattice), in such a way that a + (respectively -) spin corresponds to a normal vector pointing up (down). Following [12], we consider a bending rigidity $K$, which measures the energy cost (in units of $k_{B} T$ ) of a fold between adjacent triangles, and a "magnetic" symmetry-breaking field $h$, conjugate to the normal vectors. Furthermore, in order to study the crossover from the Ising model to the pure folding model, we shall allow the spins around a hexagon to violate the local constraint

$$
\sum_{i \in \text { hexagon }} s_{i}=0 \bmod 3
$$

(only 0,3 or 6 minus spins allowed in a hexagon), which was introduced in [10]. When the constraint Eq. 11 is verified, the model can be expressed as a 11-vertex model [10]. In our 
generalized folding model, a violation of the constraint Eq. 1 will cost an energy $L>0$, such that $L=0$ corresponds to the ordinary Ising model, while in the limit $L \rightarrow \infty$ one recovers the pure folding model. We are thus led to consider the following hamiltonian:

$$
-\frac{\mathcal{H}}{k_{B} T}=K \sum_{\langle i j\rangle} s_{i} s_{j}+h \sum_{i} s_{i}+L \sum_{\text {hexagons }} \delta\left(\left\{s_{i}\right\}_{i \in \text { hexagon }}\right)
$$

the first sum is over nearest neighbors and $\delta$ is a function equal to 1 only when the constraint Eq. [1 is satisfied and zero otherwise; the hamiltonian above will be studied in the hexagon approximation of the cluster variation method.

The CVM, in its modern formulation [14, 15], is based on the minimization of a free energy density functional which is obtained by a truncation of the cluster (cumulant) expansion of the corresponding functional appearing in the exact variational formulation of statistical mechanics. In the hexagon approximation for the honeycomb lattice the largest clusters appearing in the expansion are the hexagons, and the approximate free energy density one has to minimize, determined according to [14, has the form

$$
\begin{aligned}
f\left(\rho_{6}\right)= & -\frac{3}{2} K \operatorname{Tr}\left(s_{1} s_{2} \rho_{2}\left(s_{1}, s_{2}\right)\right)-h \operatorname{Tr}\left(s_{1} \rho_{1}\left(s_{1}\right)\right)-\frac{1}{2} L \sum^{\prime} \rho_{6}\left(\left\{s_{i}\right\}\right) \\
& +\frac{1}{2} \operatorname{Tr}\left(\rho_{6} \ln \rho_{6}\right)-\frac{3}{2} \operatorname{Tr}\left(\rho_{2} \ln \rho_{2}\right)+\frac{1}{2} \operatorname{Tr}\left(\rho_{1 A} \ln \rho_{1 A}\right)+\frac{1}{2} \operatorname{Tr}\left(\rho_{1 B} \ln \rho_{1 B}\right) \\
& +\lambda\left(\operatorname{Tr} \rho_{6}-1\right),
\end{aligned}
$$

where $\operatorname{Tr}$ stands for trace, $\sum^{\prime}$ denotes summation over the hexagon configurations that satisfy the constraint Eq. 1, $\lambda$ is a Lagrange multiplier which ensures the normalization of $\rho_{6}$ (normalization of the site and pair density matrices follows by definition, see below) and $\rho_{1 A(B)} \equiv \rho_{1 A(B)}\left(s_{1}\right), \rho_{2} \equiv \rho_{2}\left(s_{1}, s_{2}\right)$ and $\rho_{6} \equiv \rho_{6}\left(s_{1}, s_{2}, s_{3}, s_{4}, s_{5}, s_{6}\right)$ are the site, pair and hexagon density matrices respectively. The spin configuration of each cluster can be used as 
a label for the corresponding density matrix, because, as for any classical model, the density matrices are diagonal.

In writing Eq. 3 we have introduced two site density matrices, $\rho_{1 A}$ and $\rho_{1 B}$, corresponding to the two interpenetrating sublattices in which the honeycomb lattice can be divided, which will be needed to investigate the case of antiferromagnetic coupling $K<0$. These density matrices can be defined as partial traces of the pair matrix, as follows

$$
\begin{aligned}
& \rho_{1 A}\left(s_{A}\right)=\sum_{s_{B}} \rho_{2}\left(s_{A}, s_{B}\right) \\
& \rho_{1 B}\left(s_{B}\right)=\sum_{s_{A}} \rho_{2}\left(s_{A}, s_{B}\right),
\end{aligned}
$$

adopting the convention that the first spin in the argument of $\rho_{2}$ always belongs to sublattice $A$. With this assumption, $\rho_{2}$ can be defined as a (symmetrized, for convenience) partial trace of $\rho_{6}$ by

$$
\begin{aligned}
\rho_{2}\left(s_{A}, s_{B}\right)= & \frac{1}{6} \sum_{s_{A}^{\prime}, s_{A}^{\prime \prime}, s_{B}^{\prime}, s_{B}^{\prime \prime}}\left[\rho_{6}\left(s_{A}, s_{B}, s_{A}^{\prime}, s_{B}^{\prime}, s_{A}^{\prime \prime}, s_{B}^{\prime \prime}\right)+\rho_{6}\left(s_{A}^{\prime}, s_{B}, s_{A}, s_{B}^{\prime}, s_{A}^{\prime \prime}, s_{B}^{\prime \prime}\right)\right. \\
& +\rho_{6}\left(s_{A}^{\prime}, s_{B}^{\prime}, s_{A}, s_{B}, s_{A}^{\prime \prime}, s_{B}^{\prime \prime}\right)+\rho_{6}\left(s_{A}^{\prime}, s_{B}^{\prime}, s_{A}^{\prime \prime}, s_{B}, s_{A}, s_{B}^{\prime \prime}\right) \\
& \left.+\rho_{6}\left(s_{A}^{\prime}, s_{B}^{\prime}, s_{A}^{\prime \prime}, s_{B}^{\prime \prime}, s_{A}, s_{B}\right)+\rho_{6}\left(s_{A}, s_{B}^{\prime}, s_{A}^{\prime \prime}, s_{B}^{\prime \prime}, s_{A}^{\prime}, s_{B}\right)\right]
\end{aligned}
$$

where the spins in the argument of $\rho_{6}$ follow each other counterclockwise in the hexagon, and the first one is on the $A$ sublattice. In terms of the site and pair density matrices one can easily define the uniform and staggered order parameters

$$
\begin{aligned}
M & =\frac{1}{2}\left[\operatorname{Tr}\left(s \rho_{1 A}(s)\right)+\operatorname{Tr}\left(s \rho_{1 B}(s)\right)\right] \\
M_{S} & =\frac{1}{2}\left[\operatorname{Tr}\left(s \rho_{1 A}(s)\right)-\operatorname{Tr}\left(s \rho_{1 B}(s)\right)\right]
\end{aligned}
$$

and the nearest neighbor correlation function $c=\operatorname{Tr}\left(s_{A} s_{B} \rho_{2}\left(s_{A}, s_{B}\right)\right)$. 
With the definitions above our free energy can be regarded as a function of $\rho_{6}$ only and taking the derivatives with respect to the generic element of $\rho_{6}$ we find, after some algebraic manipulation, the stationarity conditions

$$
\begin{aligned}
\rho_{6}\left(s_{1}, s_{2}, s_{3}, s_{4}, s_{5}, s_{6}\right)= & \exp \left[-\lambda+\frac{K}{2} \sum_{i=1}^{6} s_{i} s_{i+1}+\frac{h}{3} \sum_{i=1}^{6} s_{i}+L \delta\left(\left\{s_{i}\right\}_{i \in \text { hexagon }}\right)\right] \\
& \times\left[\rho_{2}\left(s_{1}, s_{6}\right) \rho_{2}\left(s_{1}, s_{2}\right) \rho_{2}\left(s_{3}, s_{2}\right) \rho_{2}\left(s_{3}, s_{4}\right) \rho_{2}\left(s_{5}, s_{4}\right) \rho_{2}\left(s_{5}, s_{6}\right)\right]^{1 / 2} \\
& \times\left[\rho_{1 A}\left(s_{1}\right) \rho_{1 B}\left(s_{2}\right) \rho_{1 A}\left(s_{3}\right) \rho_{1 B}\left(s_{4}\right) \rho_{1 A}\left(s_{5}\right) \rho_{1 B}\left(s_{6}\right)\right]^{-1 / 3}
\end{aligned}
$$

where $s_{7} \equiv s_{1}$ and $\lambda$ has to be determined by solving the normalization condition $\operatorname{Tr} \rho_{6}=1$. This set of equations, together with the definitions Eqs. 1 and 5 is known as the natural iteration method [13], because it can be solved by simply iterating the equations above, and always converges to a local minimum of the approximate free energy. To find the global minimum it is therefore enough to start the iteration with different sets of initial conditions, one for each of the expected phases.

It can be easily recognized that the elements of $\rho_{6}$ are not all independent, since when two spin configurations are related by simmetry (rotation and/or reflection) the corresponding elements are degenerate. In Tab. 1 we have listed the 20 independent (up to normalization) configurations, together with their multiplicities, for the most general case, corresponding to the antiferromagnetic phase (see below). In the ferromagnetic phase we have sublattice invariance, which implies (using the same symbol for a configuration and its density)

$$
z_{3}=z_{4}, \quad z_{5}=z_{6}, \quad z_{9}=z_{10}, \quad z_{11}=z_{12}, \quad z_{15}=z_{16}, \quad z_{17}=z_{18}, \quad z_{19}=z_{20} .
$$

In the disordered phase, and for $h=0$, we have also invariance under spin inversion, from 
which one can derive the additional relations

$$
z_{1}=z_{2}, \quad z_{3}=z_{5}, \quad z_{7}=z_{8}, \quad z_{9}=z_{11}, \quad z_{13}=z_{14} .
$$

Finally, in the limit $L \rightarrow \infty$ (pure folding), the densities corresponding to configurations which violate the constraint Eq. 1 vanish, i.e. $z_{i}=0, \quad i=3,4, \ldots 14$.

\section{Results}

In this section we present our results for the phase diagram of the model Eq. 2 .

First of all, we consider the case $L=\infty, K=h=0$, studied in [9, 10, 11, 18]. For $L=\infty, h=0$ and unbroken spin-flip symmetry the only non-vanishing elements of $\rho_{6}$ are $z_{1}=z_{2}, z_{15}=z_{16}, z_{17}=z_{18}$ and $z_{19}=z_{20}$. Then the stationarity condition Eq. 7 can be solved exactly, yielding

$$
\begin{aligned}
z_{19} & =\left[2\left(\alpha^{3}+3 \alpha^{2}+6 \alpha+1\right)\right]^{-1}, \\
z_{1} & =\alpha z_{15}=\alpha^{2} z_{17}=\alpha^{3} z_{19}, \\
\alpha & =\frac{2-u-\sqrt{3-u-u^{2}}}{u-1}, \quad u=\exp (2 K) .
\end{aligned}
$$

For $K=0$ this reduces to $8 z_{1}=4 z_{15}=2 z_{17}=z_{19}=4 / 39$, corresponding to an entropy per

site $s=\ln q$ with $q=\sqrt{13} / 3 \simeq 1.2019$, whereas the exact result [11] is $q=\frac{\sqrt{3}}{2 \pi} \Gamma\left(\frac{2}{3}\right)^{3 / 2} \simeq$ 1.2087, and to a negative (antiferromagnetic) nearest neighbor correlation $c=-1 / 3$, for which no exact result is available. The very good agreement (within $0.6 \%$ ) of our estimate of the entropy with the exact value gives us confidence in applying the cluster variation method to the present model. 
Let us now turn to the analysis of the phase diagrams in the plane $K, h$ at different values of $L$. They are symmetric with respect to the axis $h=0$ and it is sufficient to describe them at positive values of $h$.

In Fig. 1 it is shown the phase diagram of the pure folding problem $(L=\infty)$. At sufficiently large values of $h$ the flat phase with $M \equiv 1$ is always stable. It is remarkable that the CVM always yields $M$ exactly equal to 1 in this phase, as conjectured in [12]. This result is presumably exact, since, as observed in [10], the flat (ferromagnetic) ground state has no local excitations. This is no longer true, of course, for finite values of $L$.

The two flat phases with magnetization $M= \pm 1$ coexist at $h=0$ for $K \geq K_{0}^{c r}(\infty)=$ 0.1013 , to be compared with the value $K=0.11 \pm 0.01$ of [12]. This first order transition point can be obtained by requiring that the free energy densities of the ordered and disordered phase take the same value, i.e. by solving the equation $f_{\mathrm{ORD}}(K)=f_{\mathrm{DIS}}(K)$. Here $f_{\mathrm{ORD}}(K)=-3 K / 2$ is the (presumably exact) free energy density of the ordered phase, as in [12], obtained by observing that this phase has vanishing entropy and neglecting the diverging $L$ contribution, which is common to both phases. On the other hand, by substituting the disordered phase density matrix elements Eq. 10 in the expression for the free energy we obtain

$$
\begin{aligned}
f_{\mathrm{DIS}}(K)= & -\frac{3}{2} K \frac{\alpha-u}{\alpha+u}+\frac{1}{2} \ln z_{19}+3 z_{19}\left(\alpha^{3}+2 \alpha^{2}+2 \alpha\right) \ln \alpha+ \\
& -3 \mathcal{L}\left[\frac{\alpha}{2(\alpha+u)}\right]-3 \mathcal{L}\left[\frac{u}{2(\alpha+u)}\right]-\ln 2,
\end{aligned}
$$

where $\mathcal{L}(x)=x \ln x$.

At smaller values of $K$, the coexistence line at $h=0$ separates into two branches where the disordered folded phase with $M \approx 0$ coexists with the flat phases. The intersection of 
the upper branch with the axis $K=0$ is at $h=0.18495 \pm 0.00005$ to be compared with the value $h=0.189$ found in [12]. In the disordered phase $M$ vanishes only at $h=0$, but this might be a consequence of our approximation (see also the discussion in the next section).

Then, at sufficiently negative value of $K$, there is a critical transition between the disordered folded phase and a folded antiferromagnetic phase with staggered order parameter $M_{S} \neq 0$ and $M \approx 0$. This transition is represented by the almost vertical broken line of Fig. 1 , which intersects the horizontal axis at $K=-0.284$. This completes the phase diagram shown in Fig. 6 of [12].

In order to understand how this phase diagram evolves while the constraint is relaxed we begin by plotting in Fig. 2 (in the $L, K$ plane) the transition line $K_{0}^{c r}(L)$ which separates, at $h=0$, the disordered folded phase with $M=0$ and the flat phases with $M \neq 0$. At large values of $L$ the transition is first-order and the curve tends to the asymptotic value $K_{0}^{c r}(\infty)=0.1013$. At small values of $L$ the transition is critical as in the Ising model $(\mathrm{L}=0)$. At $L=1.359, K=0.3038$ there is a tricritical point where the transition changes its behaviour. In our approximation scheme the critical line and the tricritical point are obtained as the solution of suitable analytical equations [19]. The derivation of these equations is almost straightforward but quite cumbersome, and we have omitted it.

The topology of the phase diagram in the plane $K, h$ remains the same until the value $L_{\mathrm{b}}=1.75 \pm 0.05$ is reached. For smaller values of $L$, in the range $1.359<L<L_{\mathrm{b}}$, the typical phase diagram is shown in Fig. 3, where the particular value $L=1.6$ has been chosen. The difference with the case $L=\infty$ is that now the first-order transition lines between the disordered and the flat phase are interrupted in some range. The two critical 
points limiting the upper and the lower branch of the interrupted first-order line (at positive $h$ ) have respectively coordinates $K=0.175, h=0.076$ and $K=0.262, h=0.0009$.

When $L$ still decreases, the branches at smaller $|h|$ (see the inset of Fig. 3) become shorter and shorter until they collapse on the axis $h=0$ at the tricritical point of Fig. 2 $(\mathrm{L}=1.359)$. In this situation the phase diagram assumes the topology of Fig. $4(L=1.3)$, with the two surviving branches separating phases with different magnetizations.

By diminishing further the value of $L$ the branches of Fig. 4 become less pronounced and at $L=1.1$ they are not distinguishable anymore. Therefore, on the transition line limiting the antiferromagnetic phase, two tricritical points (in symmetrical positions with respect to the $K$ axis) separate the first-order behaviour at more negative values of $K$ from the critical behaviour close to the $K$ axis. At $L=1.1$ the coordinates of the tricritical point at $h>0$ are $K=-0.6198, h=1.795$. These tricritical points move towards larger values of $|h|$ when $L$ decreases. It is not clear from our calculations if these points disappear at a singular value of $L$ or if they move continuously towards $|h|=\infty$ when $L \rightarrow 0$. At $L=0$ the standard phase diagram of the Ising model in a magnetic field is recovered with a critical transition always limiting the antiferromagnetic phase [17]. This phase diagram is shown in Fig. 5. The intersection of the critical line with the axis $h=0$ is at $K=-0.6214$ which has to be compared with the exact critical Ising coupling on the hexagonal lattice given by $\left|K_{\mathrm{c}}\right|=\frac{1}{2} \ln (\sqrt{3}+2) \simeq 0.6585$ 20]. The point $K=K_{0}^{c r}(0)=0.6214$ is the usual ferromagnetic Ising critical point. 


\section{Discussion and conclusions}

In this paper we have studied the phase diagram of a constrained Ising spin model describing a network of equilateral triangles embedded in a $2 d$ space. The constraint is due to the fact that during the folding the local connectivity properties of the network have to be preserved. This study has been done by applying the cluster variation method to the hamiltonian defined in Eq. 2. In Eq. 2 the term proportional to $L$ favours the spin configurations verifying the constraint which are the only surviving in the limit $L \rightarrow \infty$. In order to study networks with defects consisting of endpoints in the folding lines, we have also studied the model Eq. 2 at finite values of $L$ by progressively relaxing the constraint until to consider the case $L=0$ corresponding to the usual Ising model.

Our results can be summarized in this way. In the case of the pure folding problem we first observe the good agreement between our evaluation of the entropy $s=\ln q$ with $q \simeq 1.2019$ at $K=0, h=0$ and the exact result $q=1.2087$ of 11 . We also predict for the nearest neighbor correlation at $K=0, h=0$ the value $c=-1 / 3$. Results concerning other regions of the phase diagram in the plane $K, h$ are also in good agreement with results obtained in [12]. We have completed the results of [12] by studying the phase diagram of the model Eq. 2 also for negative values of the bending rigidity $K$. A critical transition between the disordered folded phase and a folded phase with antiferromagnetic order has been found. The critical value of $K$ for this transition at $h=0$ is $K=-0.284$. We conclude the discussion of the pure folding case making some comments about the hypothesis advanced in [12] that $M$ always equals 1 in the ordered phase and 0 in the disordered phase. Our results confirm that $M \equiv 1$ in the ordered phase, a result which is almost certainly exact, but yield $M=0$ 
in the disordered phase only for $h=0$, while close to the transition with the flat phase we have $M \sim 10^{-2}$. An order parameter of the same order of magnitude can be obtained by extrapolating with standard methods (Shanks transform, alternating $\epsilon$-algorithm and Padé approximants [21, 22]) the results from the transfer matrix method proposed in [12], obtained with strip widths in the range 2 to 8 . It would be very interesting to see how the order parameter varies when one considers larger clusters for the CVM or larger strips in the transfer matrix method, but this is beyond the purpose of the present paper. In our opinion, however, this issue cannot be settled numerically in a definitive way, and a rigorous argument would be welcome.

At finite $L$, the evolution of the phase diagram from $L \rightarrow \infty$ to $L=0$ is shown in Figs. 1,3-5. It is curious to observe the analogies between the antiferromagnetic region of these phase diagrams and the phase diagrams appearing in the study of the Ising spin model for metamagnets [17]. The hamiltonian of the Ising spin model for metamagnets includes an antiferromagnetic coupling $J$ between nearest neighboring sites, a ferromagnetic coupling $J^{\prime}$ between next-to-the-nearest neighboring sites and an external magnetic field. When the ratio $\epsilon=J / J^{\prime}$ is in the range $0<\epsilon<3 / 5$, the phase diagram of the metamagnet is similar for example to that of Fig. 4, but with the first-order branches inside the antiferromagnetic phase. The branches terminate at critical points where two antiferromagnetic phases with different net magnetizations cease to coexist [17]. These analogies can be understood by observing that the constraint energy proportional to $L$ includes an effective next-to-thenearest neighboring site interaction in the hexagonal lattice.

In conclusions, we observe that in this paper the CVM has been applied to a vertex 
model giving results in excellent agreement with numerical and exact previously known results. This is encouraging for applying the CVM to the study of other vertex models or colouring problems. In particular, in [23] an extension of the model of [9, 10, 12] which describes a possible embedding of a triangular network in a $3 d$ space has been formulated as a 98-vertex model. We think that the phase diagram of the model of [23] could be efficiently studied by applying the CVM, and work is in progress along these lines. 


\section{References}

[1] For an introduction to the subject see, e.g., Statistical Mechanics of Membranes and Surfaces, edited by D.R. Nelson, T. Piran, and S. Weinberg, (World Scientific, Singapore, 1989).

[2] Y. Kantor and D.R. Nelson, Phys. Rev. Lett. 57, 2774 (1987); Phys. Rev. A 36, 4020 (1987).

[3] D.R. Nelson and L. Peliti, J. Phys. (France) 48, 1085 (1987).

[4] M. Paczuski, M. Kardar, and D.R. Nelson, Phys. Rev. Lett. 60, 2638 (1988).

[5] F. David and E. Guitter, Europhys. Lett. 5, 709 (1988).

[6] J.A. Aronovitz and T.C. Lubensky, Phys. Rev. Lett. 60, 2634 (1988); M. Paczuski and M. Kardar, Phys. Rev. A 39, 6086 (1989).

[7] G. Gonnella and A. Maritan, J. Phys. A: Math. Gen. 24, 1987 (1991).

[8] M. Baig, D. Espriu and J. Wheater, Nucl. Phys. B 314, 587 (1989); R. Renken and J. Kogut, Nucl. Phys. B 342, 753 (1990); R. Harnish and J. Wheater, Nucl. Phys. B 350, 861 (1991); J. Wheater and P. Stephenson, Phys. Lett. B 302, 447 (1993).

[9] Y. Kantor and M.V. Jarić, Europhys. Lett. 11, 157 (1990).

[10] P. Di Francesco and E. Guitter, Europhys. Lett. 26, 455 (1994).

[11] R.J. Baxter, J. Math. Phys. 11, 3 (1970). 
[12] P. Di Francesco and E. Guitter, Phys. Rev. E 50, 4418 (1994).

[13] R. Kikuchi, J. Chem. Phys. 60, 1071 (1974).

[14] G. An, J. Stat. Phys. 52, 727 (1988).

[15] T. Morita, J. Stat. Phys. 59, 819 (1990).

[16] See, e.g., R. Kikuchi, Phys. Rev. 81, 988 (1951); S.K. Aggarwal and T. Tanaka, Phys. Rev. B 16, 3963 (1977); J.M. Sanchez and D. de Fontaine, Phys. Rev. B 17, 2926 (1978); A. Pelizzola, Physica A 211, 107 (1994).

[17] J.M. Kincaid and E.G.D. Cohen, Phys. Rep. C 22, 57 (1975).

[18] For the calculation of the entropy of the three-colouring problem of the edges of the hexagonal lattice, see also G.M. Cicuta and A. Pavone, J. Phys. A: Math. Gen. 22, 4921 (1989).

[19] See, e.g., C. Buzano and A. Pelizzola, Physica A 189, 333 (1992).

[20] See, e.g., A. Hintermann, H. Kunz and F.Y. Wu, J. Stat. Phys. 19, 623 (1978).

[21] M.N. Barber, in Phase transitions and Critical Phenomena, vol. 8, ed. C. Domb and J.L. Lebowitz (Academic, London, 1983).

[22] A.J. Guttmann, in Phase transitions and Critical Phenomena, vol. 13, ed. C. Domb and J.L. Lebowitz (Academic, London, 1989).

[23] M. Bowick, P. Di Francesco, O. Golinelli and E. Guitter, preprint T95/019, SU-4240602 , cond-mat/9502063. 


\section{Figure Captions}

Fig. 1: Phase diagram of the pure folding problem $(L=\infty)$. The solid and the broken lines are respectively coexistence and critical lines.

Fig. 2: The transition line separating at $h=0$ the disordered folded phase $(M=0)$ from the flat phases $(M= \pm 1)$. The solid and the broken lines are respectively first-order and critical lines. The tricritical point is marked by a full circle.

Fig. 3: Phase diagram of the folding model at $L=1.6$. The solid and the broken lines are respectively coexistence and critical lines. The full circles represent critical points (their coordinates are given in Section 3). In the inset it is reported the magnification of the region of the phase diagram in the empty square.

Fig. 4: Phase diagram of the folding model at $L=1.3$. The solid and the broken lines are respectively coexistence and critical lines. The full circles represent critical points; their coordinates are: $K=-0.1382, h=0.66 ; K=-0.1382, h=-0.66 ; K=0.31377, h=0$.

Fig. 5: Phase diagram of the pure Ising model on a honeycomb lattice $(L=0)$. The solid and the broken lines are respectively coexistence and critical lines. The full circle represents the usual ferromagnetic Ising critical point. 


\section{Table Caption}

Table 1: Independent hexagon configurations. 
Table 1

\begin{tabular}{|c|cccccc|c|}
\hline Configuration & $s_{1}$ & $s_{2}$ & $s_{3}$ & $s_{4}$ & $s_{5}$ & $s_{6}$ & Multiplicity \\
\hline$z_{1}$ & + & + & + & + & + & + & 1 \\
$z_{2}$ & - & - & - & - & - & - & 1 \\
$z_{3}$ & - & + & + & + & + & + & 3 \\
$z_{4}$ & + & - & + & + & + & + & 3 \\
$z_{5}$ & + & - & - & - & - & - & 3 \\
$z_{6}$ & - & + & - & - & - & - & 3 \\
$z_{7}$ & - & - & + & + & + & + & 6 \\
$z_{8}$ & + & + & - & - & - & - & 6 \\
$z_{9}$ & - & + & - & + & + & + & 3 \\
$z_{10}$ & + & - & + & - & + & + & 3 \\
$z_{11}$ & + & - & + & - & - & - & 3 \\
$z_{12}$ & - & + & - & + & - & - & 3 \\
$z_{13}$ & - & + & + & - & + & + & 3 \\
$z_{14}$ & + & - & - & + & - & - & 3 \\
$z_{15}$ & - & - & - & + & + & + & 3 \\
$z_{16}$ & + & + & + & - & - & - & 3 \\
$z_{17}$ & - & - & + & - & + & + & 6 \\
$z_{18}$ & + & + & - & + & - & - & 6 \\
$z_{19}$ & - & + & - & + & - & + & 1 \\
$z_{20}$ & + & - & + & - & + & - & 1 \\
\hline
\end{tabular}




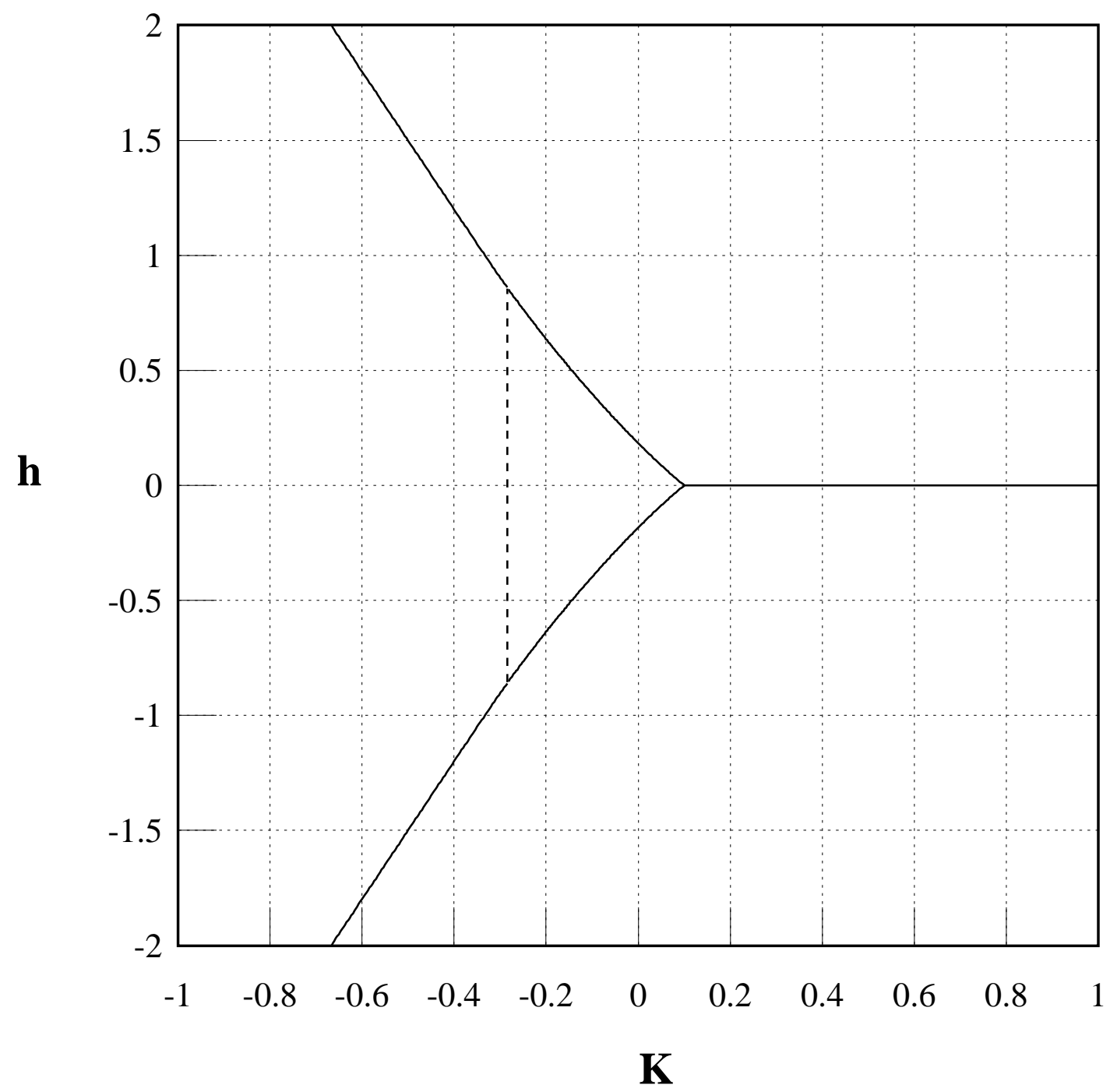

Fig. 1 - Cirillo et al. 


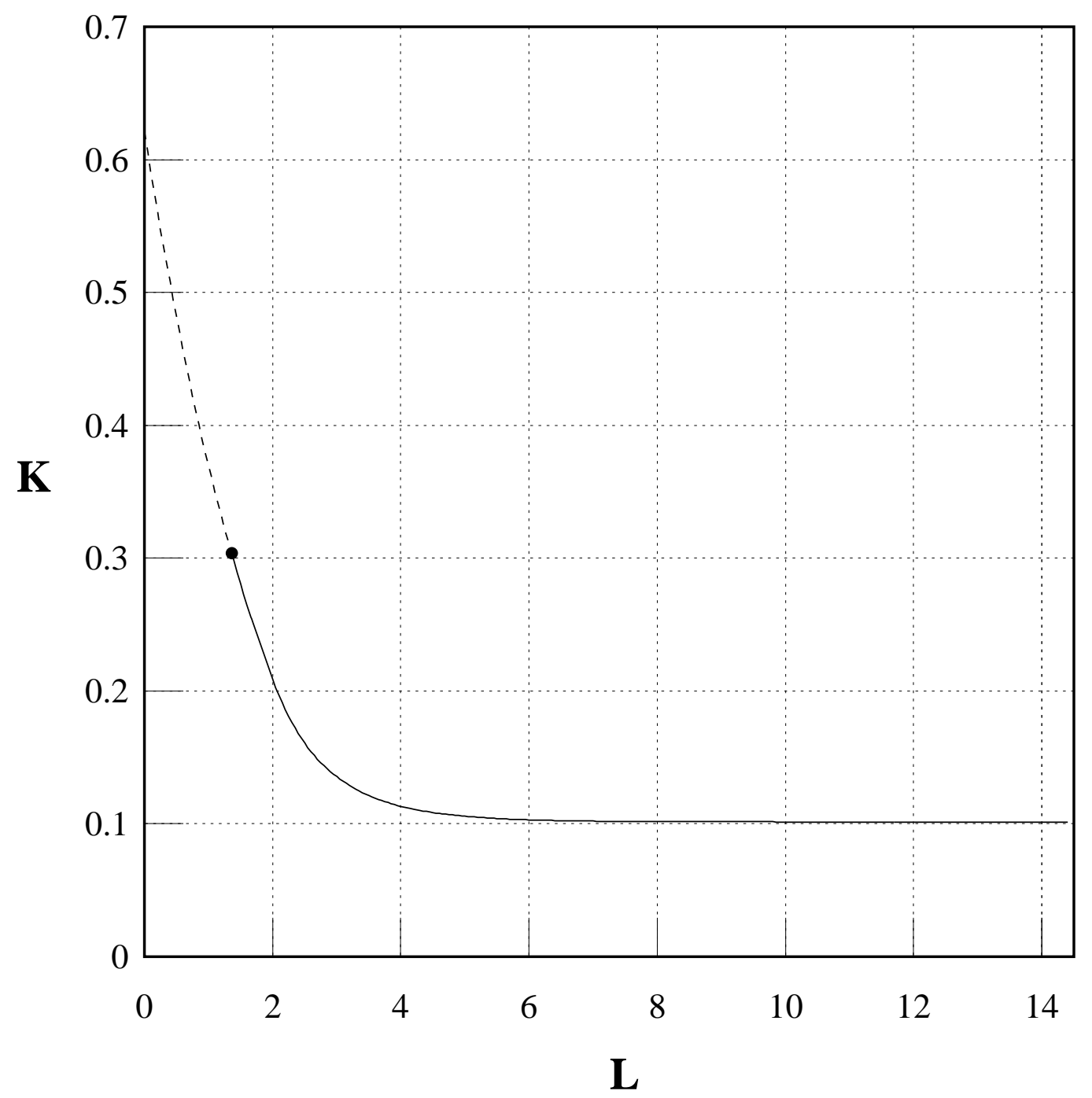

Fig. 2 - Cirillo et al. 


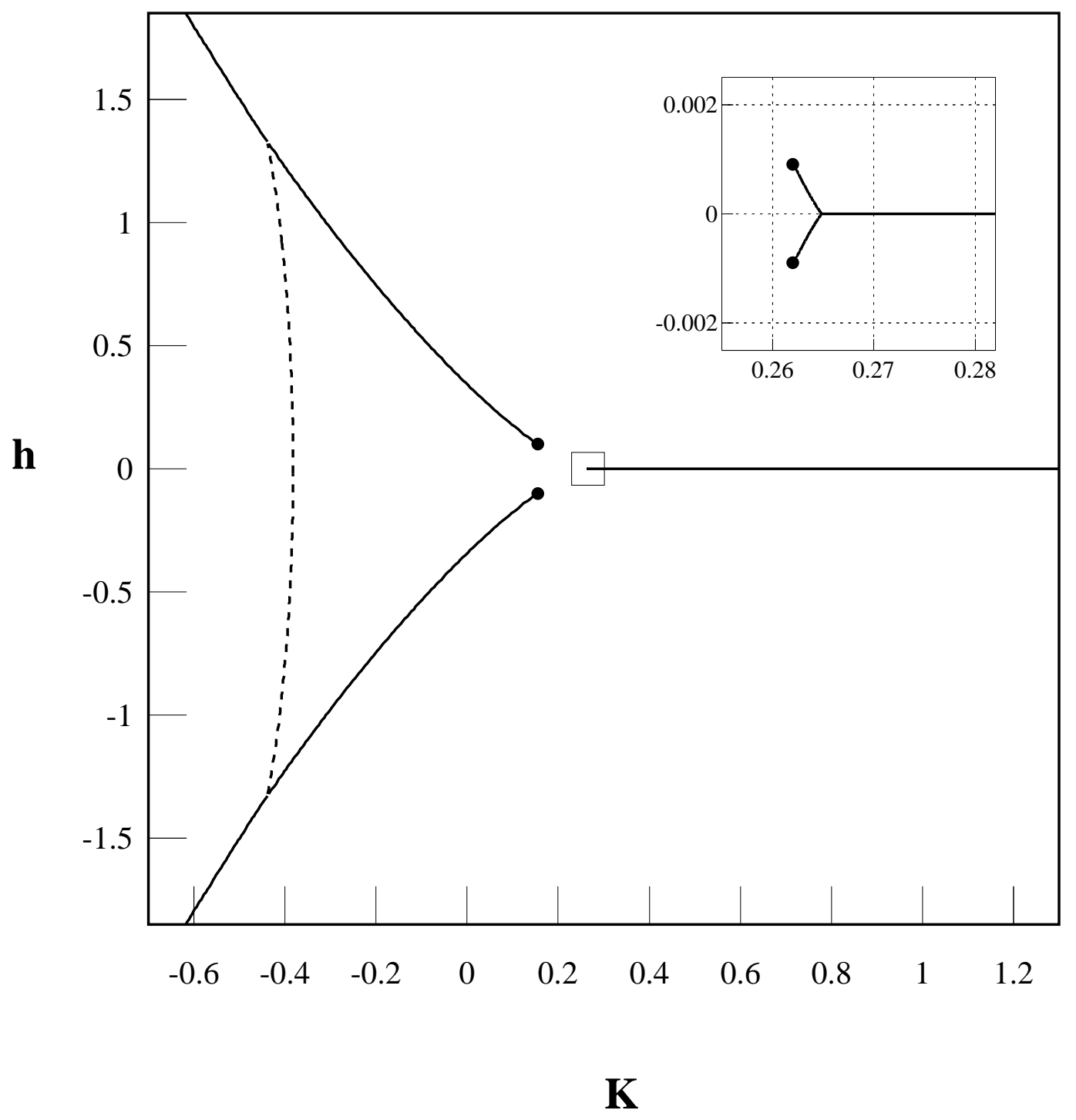

Fig. 3 - Cirillo et al. 


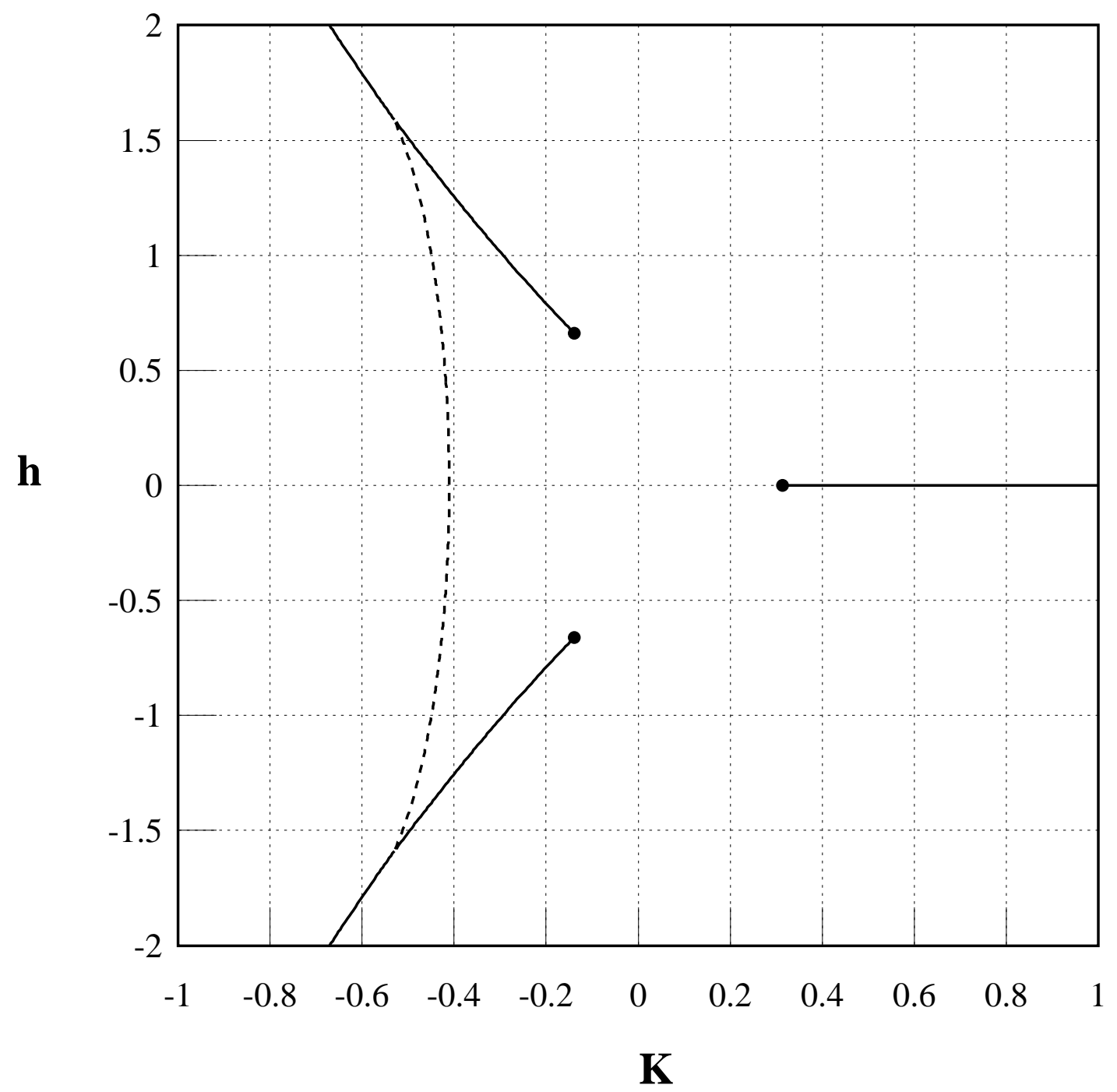

Fig. 4 - Cirillo et al. 


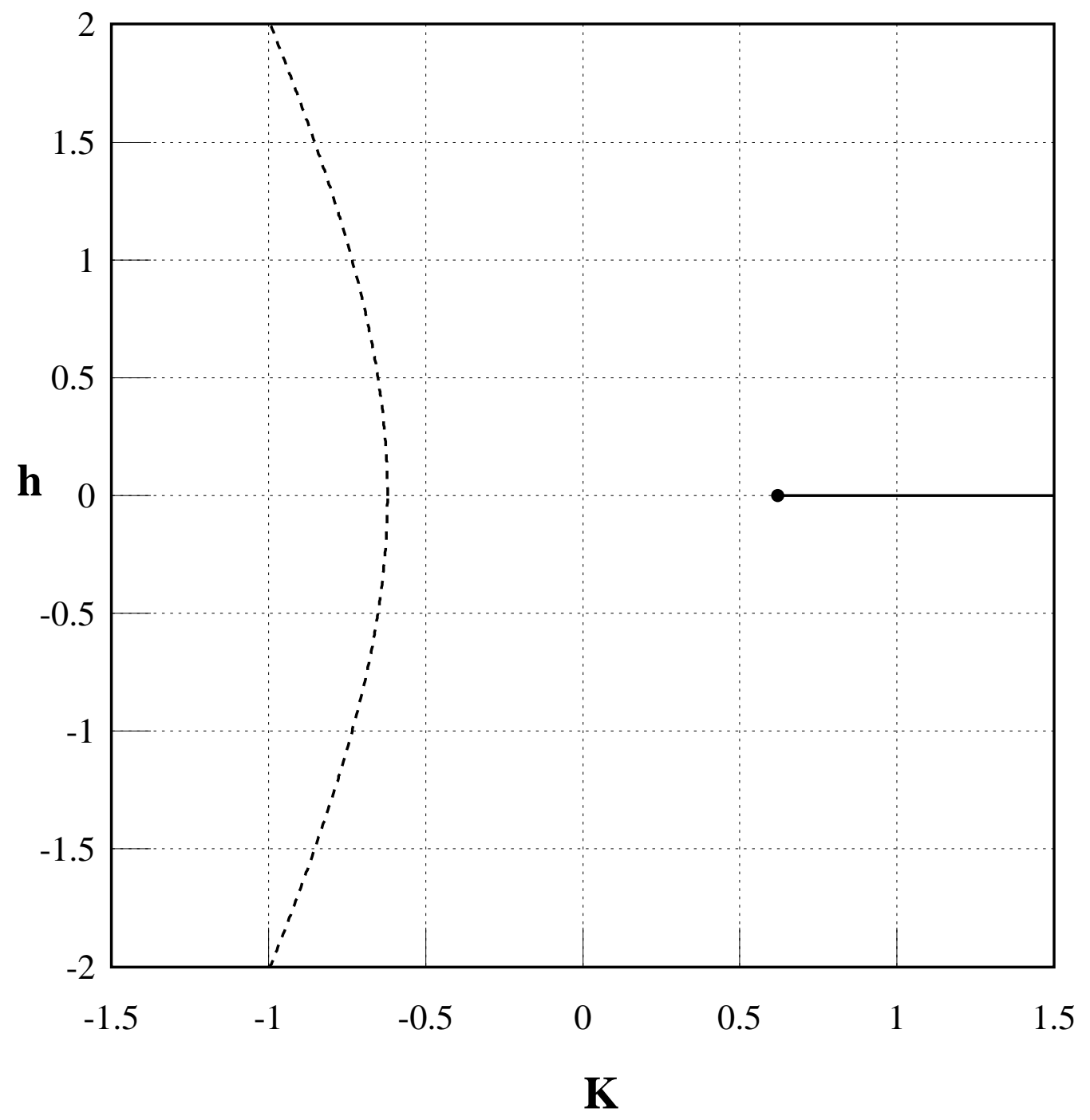

Fig. 5 - Cirillo et al. 